
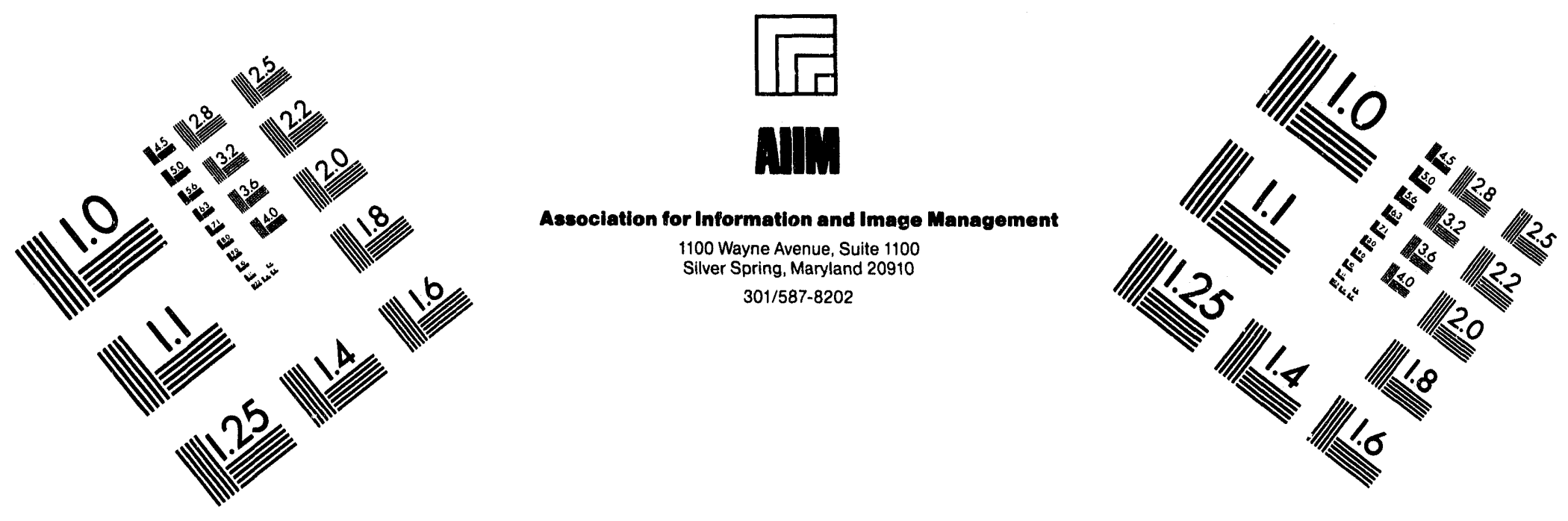

\title{
Centimeter
}

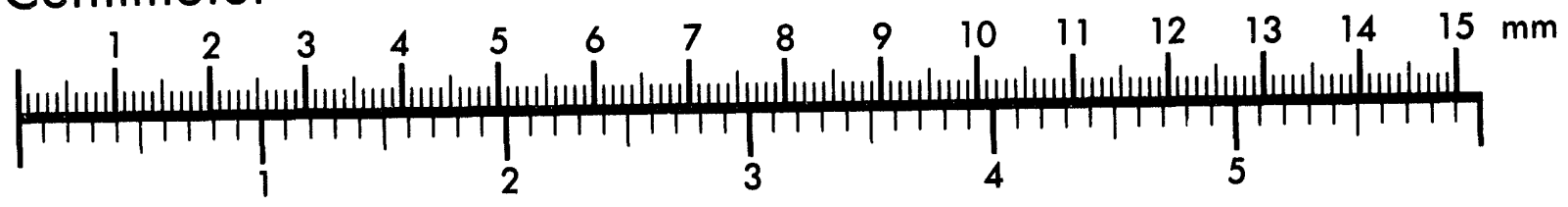
Inches
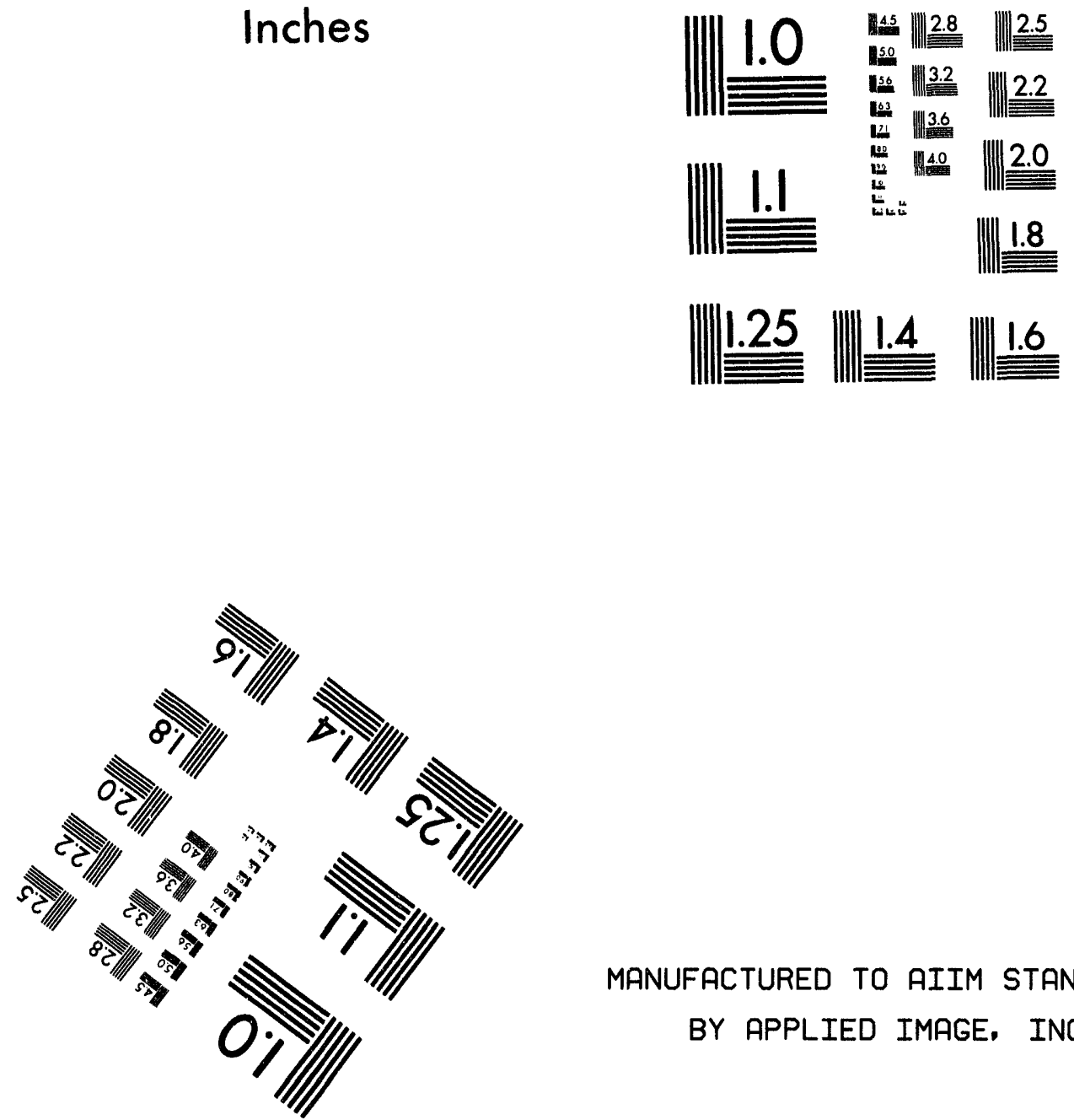

MANUFACTURED TO AIIM STANDARDS

BY APPLIED IMAGE, INC.

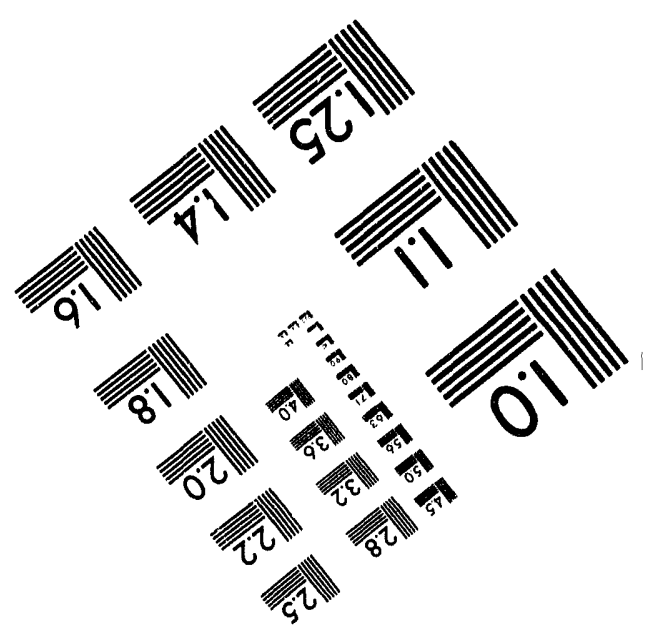



ARGONNE NATIONAL LABORATORY

9700 South Cass Avenue

Argonne, Illinois 60439

\section{GENOMIC DEFINITION OF SPECIES}

by

\section{JUL 05 B91}

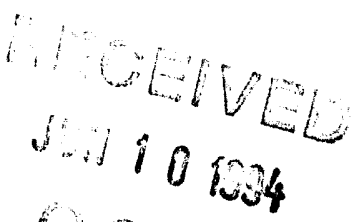

$3 \%$

Radomir Crkvenjakov ${ }^{1}$ \& Radoje Drmanac

Biological and Medical Research Division

Eor submission to

Biology \& Philosophy

July 1991

U. S. Government Durposes.

${ }^{1}$ Permanent address of authors:

Institute for Molecular Genetics and Genetic Engineering (R.C. and R.D.) and Dept. of Biology, Faculty of Sciences, Univ. of Belgrade (R.C.), P.O. Box 794, 11000 Belgrade, Yugoslavia 


\title{
GENOMIC DEPINITION OR SPECIES
}

\author{
by \\ Radomir Crkvenjakov and Radoje Drmanac
}

\section{INTRODUCTION}

For the past three hundred years philosophers and theoreticians have agreed that the question of species is one of the central open problems of biology. Darwin in the last century and Dobzhansky in this one decided to call their most influential works "The Origin of species" and "Genetics of the Origin of Species," respectively. In order to consider speciation, they had to implicitly or explicitly use their favorite species definition. However, the question of how to define species is far from settled. The issue of Biology \& Philosophy devoted to the species question (Biology \& Philosophy, 2:122-257 [1987]) is a good entry point into the current aspects of this old problem.

The species is a central theoretical notion for understanding biological diversity and evolution. The distinct aspects of looking at living systems privided by different viological disciplines resulted in a number of competing definitions. The philosophy of science has recently contributed to this debate. A species definition justifying the expectations of evolutionists and philosophers alike would have to satisfy three criteria (slightly modified from Kitcher, 1987):

UNIVERSALITY I. All organisms (evaluated over a full life cycle) must belong to exactly one species. 
UNIVERSALITY II (Uniformity). Species category is characterized by principle(s) valid for all species taxa (individual species).

ULTIMACY. The principle(s) by which one explains why a group of organisms constitute a species should also be fundamental to the explanation of biological diversity.

Currently there are two schools of thought on the subject of species definition, monists and pluralists. Monists, who include most evolutionists and some philosophers, believe there is a unique solution that meets these three criteria. Pluralists, who are mostly philosophers, hold that the living world is too complex for a single solution. The pluralists, starting from their analysis that various monist attempts at biological, evolutionary, ecological, and morphological species concepts are wanting with respect to one or more criteria, conclude that "there is no single aspect of organismal diversity that can be taken as fundamental" (Kitcher 1987). Consequently, they accept that several species definitions based on various aspects of diversity can be simultaneously true. Accepting some of this criticism, monists say that they will welcome comparison of the currently best definition with new "serious" contenders, but will not let it simply succumb to pluralist's relativization (Hull 1987).

The species question is an important component of the major theme of biological theory. It is a question of relation of biology to other natural sciences. In his illuminating book the Structure of Biological Science, A. Rosenberg (1985) has 
anaiysec =ie debate concerning this problem. Provincialists are those among biologists and philosophers who believe that stronger integration with other physical sciences is needed and that a way to achieve this goal is through more extensive theoretical developments able to transform biology from a "soft" to a "hard" science. On the other hand, autonomists hold that biology is autonomous from physics in every aspect and that is wrong to force upon biology a unity with physics. Rosenberg has shown that the dispuce between autonomists and provincialists underlies all other current ineoretical discussions in biology, and he states that neither group is fight. Provincialists correctly strive to integrate natural sciences but autonomists have been vindicated by the provincialist's failure. The triumph of the autonomists is one of fact and not of principle.

Although the molecular realm is firmly embedded in the laws of chemistry and physics, there is a vast ground between these laws and the only remaining law in biology with general validity -Darwins's law of natural selection. The complexity of biological systems has prevented the provincialists from formulating any new laws for the remainder of biology, and it is proper to consider all studies in this area as case studies without general implications.

Rosenierg concludes from his analysis that for provincialists to gain ground, new general laws need to be formulated for genetic phenomena. This is an interesting conclusion coming from the philosophy of science viewpoint, and it is devoid of the biases of practicing biologists, for molecular biologists are largely 
identified with the provincialists and population biologists with the autonomists.

The subject of this paper is the definition of species based on the assumption that genome is the fundamental level for the origin and maintenance of biological diversity. For this view to be logically consistent it is necessary to assume the existence and operation of the new law which we call genome law. Eor this reason the genome law is included in the explanation of species phenomenon presented here even if its precise formulation and elaboration are left for the future. The intellectual underpinnings of this definition can be traced to Goldschmidt (1940) and many others since. Our work shares the theoretical space with the recent contributions to evolutionary theory of Brooks \& Willey (1988), Wicken (1987), Colier (1988), and the program of Artificial Life Community (see, for instance, Langdon 1989).

We wish to explore some philosophical aspects of the definition of species in terms of the genome. The point of proposing the definition on these grounds is that any real advance in evolutionary theory has to be correct in both its philosophy and its science (Rosenberg 1985). Ghiselin (1977) divides scientific definitions in two types, one being those definitions that give "criteria whether a name is to apply to a given object or class of objects," and the other being definitions which "define concepts." Our definition deals with concepts only and requires additional specification, accumulation of genomic data, and development of more efficient genome analysis methods to be usable in sense of 
giving species "names" to groups of present-day organisms. We believe that it provides a novel view that has the potential to resolve both the monist-pluralist and autonomist-provincialist debates.

\section{DERINITION AND ASSOCIATED PRINCIPIRS}

Our proposal for the definition of a biological species is as follows:

A biological species comprises the past, present, and future, phenotypically similar and specifically adapted organisms possessing nonidentical, potentially interconvertible genomic sequences as inscriptions of the historically unique genome program generated in the process of maintenance of irreversible and inseparable cycles consisting of both inherently imperfect genomic DNA or RNA replication and organismal reproduction started by historical emergence of the first biological organism(s) as an irreducible whole consisting of a nucleic acid as a duality of the record of the material system self-reproduction program and the first structural element nonrecurrent without the organism, and of accessory material components.

The proposed definition has two parts necessary and sufficient for defining any complex entity: (1) the description of the entity's composite specificity and (2) the process (laws) for its generation. 


\section{Composite specificity}

Biological organisms are the material elements of a blological species. Species as a concept describes self-reproducing organisms and primarily represents a specific system of organization of biological material that exists for longer than the life of one organism. Existence (survival) requires compatibility with nonconstant environments. In our definition, these requirements are specified by the statement "past, present, and future phenotypically similar and specifically adapted organisms." Usually, but in theory not necessarily, more than one organism of given species lives in every section of time shorter than the life of an organism. Further, simultaneously living organisms may be physically isolated or not. This means that interaction between organisms is not a general and basic component of the definition of species, even if it is very important evolutionary factor in most species.

All biological organisms have a common ancestor, but they are phenotypically very different even within a single species. Furthermore, in many cases organisms of closely related species are more different than organisms of evolutionarily more distant species when only separate characteristics are compared. Most importantly, there is a discontinuity, i.e., an unbridgeable gap, between organisms of different species despite their common ancestor. The simultaneous existence of more than one species is recognized by phenotypical differences (morphological definition) and their adaptational meaning (ecological definition), by 
reproductive isolation and organismal interaction in the population (biological definition), or by following the discreteness relation through evolution (evolutionary, cladistic definition) (see Biology \& Philosophy, 2:122-257 (1987]).

The question is, which organizational level accounts for all of these species characteristics? Any phenotype as derived from a genome has a lesser chance to give a correct picture, but looking at genomic sequences only "phenotypically" (morphologically) is also not correct. Firstly, genomic sequences are nonidentical but similar, just as organisms are. Nevertheless, the discreteness of and evolutionary links between species can be more precisely and fundamentally defined on the basis of genomic sequence for every species, even in presence of individual differences.

The statement "nonidentical, potentially interconvertible genomic sequences" is the basis for recognizing one species from other. This means that individual genomic sequences within species can be regenerated in the process of organismal reproduction, but any individual genomic sequence of one species can not give the individual genome of other existing or extinct species. Because of this, an organism of one existing species can never give birth to an organism of another extant (or extinct) species, but only to an organism of the same species, or of a historically new species, or to inviable mutants. Reproductive isolation is a specific phenotypical consequence of this principle and is valid for most sexual species. Any vertical (mutation, deletion, duplication, translocation, transposition) or horizontal (sexual recombination, 
transfection, symbiotic merge) changes of genomic sequences in reproductive processes make contributions to the generation of sequence differences, and some of these will contribute to phenotypic organismal diversity within species or, more rarely, the creation of a new species. However, the individual characteristics of genomic DNA within species can be repetitively produced starting from different parents, clones in asexual species, etc. Interconvertibility includes reversibility but not necessarily through identical steps. Thus, due to ubiquitousness of vertical genomic changes in living forms, the horizontal changes (sex, interaction in population) are not generally necessary for organismal diversity, species integrity, and interconvertibility.

The elaborated principle of genome sequence inconvertibility can serve as a universal and, as nearly as possible, an absolute basis for species recognition. Genomic sequence interconvertibilty can be a useful parameter for future systematics as the more appropriate and more universal level for identification of discreteness and unbridgeable gaps among species. The organisms possesing genomes which can be defined as potentially interconvertible, even if reproductively isolated, or inconvertible with genomes of other organisms, even when the organisms are all phenotypically very similar, will constitute a species. For example, a reversible mutation responsible for reproductive isolation does not constitute the splitting of a species.

There are two sides of inconvertibility; probabilistic impossibility and system impossibility. For any complex material 
system, one can define physically very difficult changes or very improbable combination of changes, or, even more importantly, changes which are incompatible with existence of the system as a whole. Due to the inseparability of the evolution of the genomic sequence and the wider organized molecular system (cell, organism) of which it is a component, the intentional change of only genomic sequence or the transplantation of genome into the cell of another species will not make a viable organism of this genome's species because of genome-cell incompatibility. The multitude of simultaneous changes needed to overcome this barrier is an example of probabilistic impossibility, and sequence changes back to parental type with a lethal outcomes are an example of systemic impossibilities.

What is the theoretical framework for discreteness and uniqueness of species in the presence of individual differences? This framework is a formal, logical entity called the "genome program." Each and every organism of one species, with its particular genomic sequences and phenotypic traits, is the product of a "historically unique genome program." The program is a specific pathway of self-reproduction of a particular material system, i.e., a specimen biological organism of a species. A species genome program is the product of ancestral programs las they have evolved up to that time) and environmental constraints. This historical logic provides possibilities and constraints. The program is a framework limiting the range of individual differences, adaptational possibilities, and capacities for 
"starting" a new species. The logic of the program provides those constraints and also results in stability and long life of the species. Program and species are historically unique individuals. In spite of random changes in nucleic acids as the material record of the program, the uniqueness of the program survives as such or it is extinguished before or after giving a birth to a species that has a new program. The program includes constraints which limit changes of both the record and the whole system. The genome program consists at least of the developmental subprogram (ontogenic program), reproduction subprogram, and the inscription rule -- a specific way of making the development and reproduction program record. By the inscription rule we mean the class of phenomena of which an example is that one protein with the same time-space expression pattern can be coded in two species by genes with different numbers of introns and exons or even by genes without introns.

Definition of process for species generation

A species, i.e., phenotypically similar and adapted organisms with interconvertible genomic sequences, is not sufficiently defined if the process of its generation is not specified. In our definition, species are defined as inevitable products of the process of self-reproduction of biological organisms. The formation of inconvertible, discrete, and historical entities by self-reproducing systems in general and biological organisms in particular is the consequence of several irreducible properties of these systems which are basic components of the genome law. These 
properties are realized in the first biological organism which was formed from non-reproducing material systems. Other, derived properties can be added on in organisms formed in an uninterrupted evolutionary chain, but the basic ones cannot be lost. The basic properties are as follows:

i) the wholeness of organism which makes it distinguishable from environment and cannot be reduced to any one part of the system;

ii) the existence of a system's part which has both the property of structural element and property of material record of a formal entity-program for self-reproduction; the system both retains the capacity for self-reproduction within the range of changes of this part and its copying of this part by reproduction is allowed to be imperfect by physicochemical laws; and

iii) the system's reproduction in historically defined environment leads to existence of at least more than one organism in more than one generation.

By "historical emergence" is meant that since the basic properties cannot be attributed to the system's components per se (i.e., when they are outside the system), but only when they are within the system, the whole of the system had to emerge once at the very beginning of the process. The first system's component, which can not be regenerated outside system after emergence, but only within it with the help of other components, has the property of material record of program. The "accessory components" are helper 
components which are not necessarily formed within system (but can be) and which are internalized within system by the emergence or reproduction.

Generally imperfect reproduction leads to organisms that differ from each other, but their genomic inscriptions are interconvertible. One can ask what is required, after sufficient time has elapsed, for the inevitable occurrence of organisms so changed that they, although being able to reproduce, are unable to revert to the parental type no matter how much time is allowed for it. Or, more directly put, what is the cause for the origin of species category. There are two sides to this question. One is why there is discreteness, i.e., gaps between species. The other is why is the transformation of an organism of old species into a new one $a$ unique and irreversible event. The necessary conditions for organismal and hence species occurrences are the sufficient complexity of both systems and environments. This granted, the inevitability of species occurrences as entities separated by gaps is a consequence of the quantum, or discrete, nature of physical laws whose resultant derivative is a genome program. To one cognizant of modern physical science, the absence of a continuum of structures on any level of organization of matter requires no explanation beyond the discovery of natural laws with quantum properties.

The irreversibility and uniqueness are the result of a probability so low as to represent a virtual impossibility of reproduction in certain connecting stages through which systems 
would have to pass to both convert back and remain selfreproducing. The inseparability of rebuilding of a component with a property of being a program record from system reproducticn, i.e., replication from reproduction in biological organisms, and the inherent directionality of this coupling are a simplified notions for complex physical causes which lead to the irreversibility of speciation. Another concept is that speciation is a historical event and hence irreversible.

Both the characteristics of the first organism that emerges and the genome law which causes the origin of species are definable by consistent application of more general rules of self-reproducing material systems. The wholeness of the first biological system can be described in a light of the dual properties of the nucleic acid molecule. This molecule provides the basis for replicable program inscription through chemical complementarity. Simultaneously, as a material component, it constitutes part of the system and has a structural role, which in interaction with internalized components from environment (accessory components) forms the whole of the reproducing organism. The structural role of nucleic acids has been retained in present-day organisms, as well as the duality of structure and inscription. Only a specific nucleic acid of all that could arise in the specific moment of history had a potential of the necessary interaction with other components to form selfreproducing organism, "the irreducible whole," which started the uninterrupted chain of reproductive cycles that is called evolution. 
Nucleic acids also satisfy the obligatory property of selfreproducing systems of generally not requiring perfect record copying. Replication based on complementary pairing is not primarily sequence-specific in the sense that the fidelity of any base pair replication is independent of neighboring sequences but dependent on some structure either of the replicating enzyme or of the nucleic acid itself. Not only the structures defined by sequences are necessary for replication, but also, several different sequences can have the same derived structural element, as is evident in modern organisms. Thus, organisms based on nucleic acids can tolerate imperfect replication and are unable to avoid it. Complementary pairing opens the additional possibility of large and immediate sequence changes via recombination. Briefly, our view on the process of generation of species is that intrinsic characteristics of the first nucleic acid-based system and the complexity of environment in an uninterrupted process of imperfect replication and reproduction bring about the formation of historic and discrete entities which can neither interconvert nor arise again without external prog:am, and these are biological species.

Both the genome law of biological systems and the general law of systems with intrinsic program (self-reproducing systems) assume effects of changeable environment and the action of law of natural selection on nonidentical organisms. Furthermore, the logic of these systems is the production and maintenance of systems in spite of the action of destructive forces and in spite of the fact that 
the recurrence of formation of these systems by physicochemical forces acting alone on molecules and molecular systems on this planet is impossible. Natural selection alone (and even more when coupled with environmental changes, especially drastic ones) is the process of eliminating certain variants, thus providing additional historical constraints on further evolution. An inherent capacity of sufficiently complex, imperfectly self-reproducing systems to generate discrete, inconvertible systems is imprinted by these constraints. Some possible species will never evolve, some will be extinguished shortly after they appear. Some will never evolve in other, slightly different circumstances. During the existence of a species, organisms within the species differ in their precise modes of adaptation to surroundings. Thus, species are historically unique in part because of environment. But discreteness and inconvertibility of any species is not created and maintained, and can not be suspended, by natural selection. Instead, the complexity and high integrity of both program/record entity and self-reproducing organisms as a whole, coupled with inherently imperfect replication and reproduction, are the causes of the origin and maintenance of species as discrete and inconvertible individuals.

\section{DISCOSSION}

Our discussion of genomic definition of species is limited to showing how it conforms to the three principles. formulated by philosophers. 


\section{Oniversality I principle}

Concerning the Universality I principle that every living being belongs to exactly one species, we consider that there is only one way of looking at this principle worth pursuing: that life is based on programs. Since program is the central concept in the philosophical analysis of our definition, an attempt to define it avoiding teleological. implications is provided. While information is an unbounded quantifieable property, a program is a closed and finite information system. It is an specification of a pathway through the n-dimensional space-time manifold. Any material system is produced in the manifold by a specific pathway as a result of actions of natural laws. Complex systems are phenomenologically those subgroups of material systems for which the probability of the pathway recurring is infinitely small unless the information system to specify the pathway is available either internally or externally to a material system as a causal reference. A system's program is a formal logical entity and can be recorded by any material means to exist as a copy outside of the system simultaneously, before, or after it (Langdon 1989). Our view considers organisms as material, physical systems, self-organized in time and space according to a genomic program, the hereditary blueprint that defines in a special way properties they exhibit. In present-day organisms, the inscription of the blueprint resides in a sequence of nucleotides that make up the chromosomal DNA of cells. In protobiotic organisms, RNA molecules constituted both the inscription and most of the system. 
Biological organisms are the only natural systems built according to a program and thus they share this property with many man-made objects from stone axes to cars. Since the program of the latter systems is external, they can not be called self-reproducing. Thus, only systems with internal self-reproduction programs can be equated with organisms. However, a group of systems built according to a single program, either external or internal, is always a species.

We do not mean to imply that other kinds of programmatic systems exhibiting life-like properties are physically impossible since they have not been confirmed to have been a part of this planet's natural history. At least the possibility of artificial life based on such systems should be taken into account (Langdon 1989).

The common denominator of all organisms is the existence and inseparability of a program, its inscription in a nucleic acid sequence and a molecular system, and their common descent via reproduction from the first organism(s) having the same characteristics but generated by historical emergence. We believe that a more general statement about biology is impossible to make at this time. Other statements that have been advanced exclude one or another forms of extinct or extant life. The species is equally as basic to biology as the organism. Therefore, it is reasonable to ask to see species explained in terms of program, inscription, and system. 


\section{Oniversality II principle}

The Universality II (uniformity) principle, that it must be possible to characterize a species category by principles valid for all species taxa, has been confused with the principle of absolute operationalization of biological systematics. By this second principle is meant that once the principle(s) of uniformity are known, the additional uniform criteria based on them must exist too, able to determine the membership of an organism into species irrespective of the type of species. The absence of absolute operationalization is the property of all species definitions advanced so far. The operational criteria for a species membership are always relative and local, there is borrowing from previous assumptions about species properties in definitions purporting to define these properties de novo. We show below why this was a reasonable approach to take.

The above statement of operationality failure is not a prelude to a claim that our definition has the potential to avoid it. Quite the contrary is true. The definition on careful logic analysis will be found wanting in this respect. However the details of this failure define the major philosophical problem for systematics by the assertion that no absolutely operational species definition is possible. The principle of the impossibility of conversion of one DNA inscription of a given genome program into a DNA inscription of another genome program that has existed by a natural process has a problem in this respect. Nevertheless, the explanation of the existence of biological discontinuities visible 
as speciation events by the irreversibility of genome (inscription) changes is central to our insight. To differentiate genomic programs by this principle absolutely implies the knowledge for all species taxa of quantitative and qualitative limits which genome changes are naturally possible for all species taxa without reference to any phenotypic particulars of the organisms and species in question.

Our definition stops short of asserting that the analyses of DNA sequence of a single organism or a preset number of organisms is sufficient to know the genome program absolutely, i.e., to differentiate between the alternatives of DNA inscriptions of the same genome program and DNA inscriptions of the two or more genome programs. Although we believe this will be possible to do with a sufficient degree of accuracy for the majority of species if the preset number is reasonably high, there cannot be an assurance that no exceptions will be found. It is argued below that universal knowledge of this type is not generally possible.

The problem is an epistemiological one of the nomological kind. Names can be given to things directly, but there is a class of things which can not be recognized except by the effect they have on another thing or by the possibility that they are transformed into another thing. In that case, an exact rule of transformation must exist to allow naming the primary thing after the effect it is causing in the second thing. Black holes come to mind as examples of things named after their effects. The derived properties are distinguished from apparent derived properties by 
the fact that the set of real derived properties is necessary and sufficient given the transformation rule to discriminate the primary thing from other things. The transformation rule eliminates apparent but not real derived properties from consideration. The fact of existence of the rule provides insufficient grounds for the absolute operationality of naming definition based on the derived properties of secondary things. The understanding of the rule in, at least, a local sense is an unavoidable component of correct naming process.

Our assertion is that the species category is a class of individuals, each individual being a historically unique genome program. With respect to their individuality, it is irrelevant that each program usually exists recorded in numerous copies. Each copy has a different inscription in the form of the order of nucleotides in the DNA. Two different inscriptions can generally represent either a single or two different genome programs. The inscriptions representing one program, i.e., within species, are more similar among themselves than they are to any given inscription of another program. Due to the inseparability of the program and its inscription, there must be a rule that transforms one into the other. However, there is no reason a priori to expect the rule to be the same for all species taxa; rather, the rule is a historically frozen event valid for smaller or larger sub-groups of species (higher taxons). Therefore, the species definition based on DNA sequences or on any other derived phenotypic property can never satisfy the criterion of absolute opertionality. 
However, the genomic DNA sequence as an organizational level of the system most directly connected with the genome program must represent the basis of a definition much closer to absolute operationality than any based on the other phenotypic levels of organization, which are connected to a genome program much more indirectly and without exception via a DNA sequence.

The organisms within a species are systems which give a unique outcome -- the survival (of the program) due to the presence of a copy of the unique genome program in each of them. Therefore the exhaustive study of each of these systems as wholes gives the knowledge of the program and consequently the ability to differentiate any subsequent system as having the same or different program. Although the methodology to gain this knowledge can apply to all species taxa, the knowledge itself is particular and applies only to the species under study. This is the reason that the various empirical systematics have been more successful than the theoreticalones, since they always opted for local optimization rather than for general validity.

The Universality II (uniformity) principle is much broader than the absolute operationality principle. The uniformity principle states that the genome program, i.e, species is recognizable from analysis of the organisms (systems) which embody it and by the proximity principle, mostly from its most proximal derivation, which is the inscription in the form of a genomic DNA sequence. The general features specified above of the genome program as a transformation rule connecting genomes and organisms 
are sufficient for the definition of species category. The fact that the details of the rule vary from one group of species to the next do not preclude unification of all such groups by the general features of this rule and their distinction from any single or groups of non-species, i.e, things that can not be so described.

The new science of genomics is in its infancy. Once sufficient data about genomes are collected and analyzed in an empirical framework, the systematics theory most consistent with the reality will be discerned more readily. Because the genome level is a newly opened and unexplored level of biological organization, the species definition based on the duality of the genome program and its inscription in the DNA sequence should not be compared in the current degree of operationality with the interpretative definitions purporting to make sense of already collected biological data and information. However, the example of viruses already illustrates the natural displacment of systematics based on more derived phenotypic levels by the one based on sequences. Furthermore, the potential of sequence-based systematics to achieve the higher degree of operationality with the availability of the genomic sequence data is apparent to practitioners at the cutting edge of systematics of more complex organisms too. The theoretical reasons for this are given above in the discussion of the operationality problem.

oltimacy principle

The turning of molecular biology towards genome can be seen from the interest in various genome projects. The genome level is 
the next one up from the gene in complexity and is becoming accessible through comparative analysis of genomic sequences. It is a relatively contained level which potentially can lead to its complete material description, which is still unthinkable for other even more complex levels of biological organization. Due to these experimental developments, theory has to find a place for the genome that is different from that of a mere sum of genes. In our opinion, both Rosenberg (1985) and Wicken (1987) correctly point out that the most profitable approach for formulating adequate evolutionary theory is to consider the period of emergence of life in the primordial broth. At that time, the first biological organism had not yet evolved the complex paraphernalia necessary for today's survival. The empirical fact that RNA molecules can catalyse RNA phosphodiester bond formation led to the proposal of a protobiotic "RNA world" (Sharp 1985). Thus there is a reason now to consider only the option of RNA-based life, the first living system which had genome consisting of RNA and no energytransforming machinery. It was directly subject to natural selection. Curiously, the simultaneous theoretical treatment of genome and the emergence of first organisms seems not to have been attempted so far.

What are the arguments for proposing the ultimacy principle in terms of the genome? Since all phenomena concerning energytransforming machinery have evolved after life first emerged and are contingencies, not essentials, of living systems, it is natural that no generalizations of the type of natural law can cover these 
historically added-on phenomena that represent the majority of present-day biology. We belleve that Rosenberg is right in calliag the search of principles of organization of phenomena in this realm "case studies" -- implying these principles, once uncovered, have no general validity.

However, the current opinion (exemplified by Rosenberg) is that the gene level is the highest covered by the laws of chemistry and physics. To us this seems wrong in the sense that first living system consisted of a replicating genome and not a gene or genes. The genes came later as functional specializations. The principles of organization of this first living system are retained in all organisms and represent the only biological law generally valid besides the law of natural selection. We call it the genome law. When explicitly formulated with the principles enumerated in our definition, the genome law must require as its consequences at least i) that living systems be naturally organized into species consisting of individual organisms, ii) increasing complexity, and irreversibility of evolution, and iii) the existence of genealogical or historical constraints to variation (Drmanac and Crkvenjakov, in preparation). The ease with which molecular explanation reaches up to the present-day genes does not mean either that they existed in the first living system or that they are central to the biological explanation. It is an epistemilogical problem that biological phenomena are much easier comprehended if subdivided and studied as individual traits of an organism. However, this artificial livision for the sake of 
practicality sacrifices the chance of uncovering laws of general validity. Thus, both the gene theory and Mendelian genetics fail to "carve nature at joints" (Rosenberg 1985), since they do not explain either the individuality of organisms nor the omnipresence of species in any natural way.

Thus, while the laws already operational in the first and simplest living systems are recognizable, the real explanations for present-day species are currently beyond comprehension in their entirety. It is not that the biology is unconnectable to chemistry and physics, but we have not been capable of achieving the connection due to lack of concentration on the simplest, or even better, ideal organisms. The word "ideal" is used here to indicate the proposal to use the defined methodology borrowed from the physical chemistry. The methodology consists of definition of an ideal situation and its laws and the sequential introduction of additional assumptions consistent with, but not derivable from, the ideal case to asymptotically approach the reality of studied phenomena.

The simple questions about the ideal living system and the species it belonged to require definite answers. Since the logical edifices of different evolutionary theories can be convincingly shown to be wrong only on the evident logical errors, gedanken experiments on the ideal system able to reveal such errors are an indispensable tool. If something is wrong in the ideal system, it cannot be right for the real systems represented by it. 
The first self-reproducible RNA system was constituted with the characteristic to permanently mutate. Therefore it could not base its survival on producing exact copies. Rather, its survival must have been based on the ability to reproduce the same informatical program from nonidentical nucleotide sequence instructions. Why the same program? since there is always only a limited number of outcomes that allow self-reproduction, program stability is the inviolate condition for survival. Starting from the premise that retention or changes of genetic material is underlying evolutionary events, the only way that any change in sequence can be retained, either progressive or otherwise, is through the ability of the program to execute the self-reproduction of numerous nonidentical copies in each generation. If it were not for this informatical cohesion of individual copies, the rate of attrition due to environment would be too high for such line to exist and much less evolve.

The cause of evolution implied in this description of ideal organisms is the inevitability of rare genome change which creates a new program due to a background of incessant mutations which do not change the program. The evolving line does so usually in two steps, the last of which is obligatory. These steps are the increase in length of inscription during previous generations without compromising the execution of the program, and the contextual change which recruits the previously nonessential part of the inscription in a speciation event into the essential whole of a new program. Therefore the evolving line of ancestors and 
descendants must shield the systemic property of inscription increase against any qualitative modification, regardless of cost. The systemic properties of the program are not subject to a character selection.

The analysis of the ideal system outlined above, is a rudiment of a formal description which will account for any life-like systems based either on nucleic acids, or otherwise materialized, in necessary and sufficient terms. If accepted, this view on the origin of life leads to the priority of whole over parts, genome over genes in evolutionary events. The living systems have no purpose, nor strive to achieve any function. They exist conforming to natural laws and, while they do, survive. The irreducible requirement for survival is the complete execution of the genomic program by the system. Any way of achieving this is acceptable. Since both system and program consist of multiple components, their reaction to environment is always combinatorial; speciation is one such reaction. They try out over sufficient time all possible interactions of their components that allow both the internal execution of program and system's existence in the environment.

Our intention was to show how the genome definition of species conforms to the three principles of Kitcher (1987). Each organism belongs to exactly one species since it is a system with one and only one genome program. All species taxa belong to the species category since each has a unique genome program. Finally, the ultimacy principle is satisfied by the assertion that the genome 
level is the fundamental one for explanation of biological diversity. This is based on the analysis of the ideal case, the first organism/species proposed to have consisted of a replicating RNA molecule and accessory components. The only laws valid for it and for all living beings descended from it since are the genome law and the law of natural selection. The organism/species duality, the irreversibility of evolution, and the time-dependent increase in diversity are natural consequences of the interaction of the two laws.

Any new species definition is useful to the extent that it proposes a simplifying solution for this open scientific problem. In one of the traditions of biology, our attempt is reductionistic. By redefining the problem and putting it into new terms, its reality is made more transparent, and this in turn makes the proposed solution more susceptible to either refutation or confirmation. The new terms are very simple. Instead of ascribing the property to organize into species to the interactions between organisms, the proposed definition is based on the view that belonging to a species is an intrinsic property of organisms and that this property is genetically defined.

The way of reconciling monists and pluralists is open now. Logically, if there is a biological level fundamental to the explanation of the biological diversity, the pluralists' position of several definitions being simultaneously correct each for the particular level derived from the fundamental one need not be abandoned. Monists would be vindicated in their belief in the 
unity of biology but would have to accept that their particular favored definitions are correct only by the virtue of being supervenient on the fundamental one.

The unification of biology with other natural sciences is provided by defining biological systems as a specific type of organization of matter within the class of self-reproducing systems. Simultaneously, the specificity of biology is retained since it covers the area of extension. The physicochemical sciences are concerned with the duality of matter and energy, and the biological extension of this is the focusing on the duality relation of matter/energy complex and information, or, more precisely, the duality of material systems and their programs.

\section{ACRNOWLEDGMENTS}

We thank David E. Nadziejka for editorial assistance and Professor Nikola Tucic for leting us use his private library of evolutionary biology. An earlier version of this manuscript was completed at the Institute of Molecular Genetics and Genetical Engineering, Belgrade, Yugoslavia.

The work was supported in part by Science Fund of Serbia, Yugoslavia, U.S. Department of Energy grant DE-FG02-88ER60699, U.S.- Yugoslav Joint Board grant JF-820, and in part by the U.S. Department of Energy, Office of Health and Environmental Research, under contract No. W-31-109-Eng-38. 


\section{RETERENCES}

Brooks, D.R. and E.O. Wiley, Evolution as Entropy: Toward the Unified Theory of Biology, Univ. Chicago Press, 2nd ed., Chicago

Colier, J.: 1988, "The Dynamics of Biological Order" in Entropy, Information and Evolution: New Perspective on Physical and Biological Evolution, eds. B. Weber, D.J. Depew and J.D. Smith, MIT Press, Cambridge, 227-242

Ghiselin, M.T.: 1987, " Species Concepts, Individuality, and Objectivity", Biology \& Philosophy 2 127-144

Goldschmidt, R., 1940, The Material Basis of Evolution, Yale University Press, New Haven

Hull, D.A.: 1987, "Genealogical Actors in Ecological Roles", Biology \& Philosophy 2 168-183

Kitcher, P.: 1987, "Ghostly whispers: Mayr, Ghiselin and the "Philosophers" on the Ontological Status of Species", Biology \& Philosophy 2 184-191

Langdon, C.A.: 1989, "Artificial Life", in Artificial Life, ed.

C.A. Langdon, Addison-Wesley, Reading, Mass., 1-48 Rosenberg, A., 1985, The Structure of Biological Science, Cambridge University Press, Cambridge, England Sharp, P., 1985, "On the Origin of RNA Splicing and Introns", Cel1 $12 \quad 397-400$ 
Wicken, J.S., 1987, Evolution, Thermodynamics, and Information: Extending the Darwinian Program, Oxford University Press, Oxford 

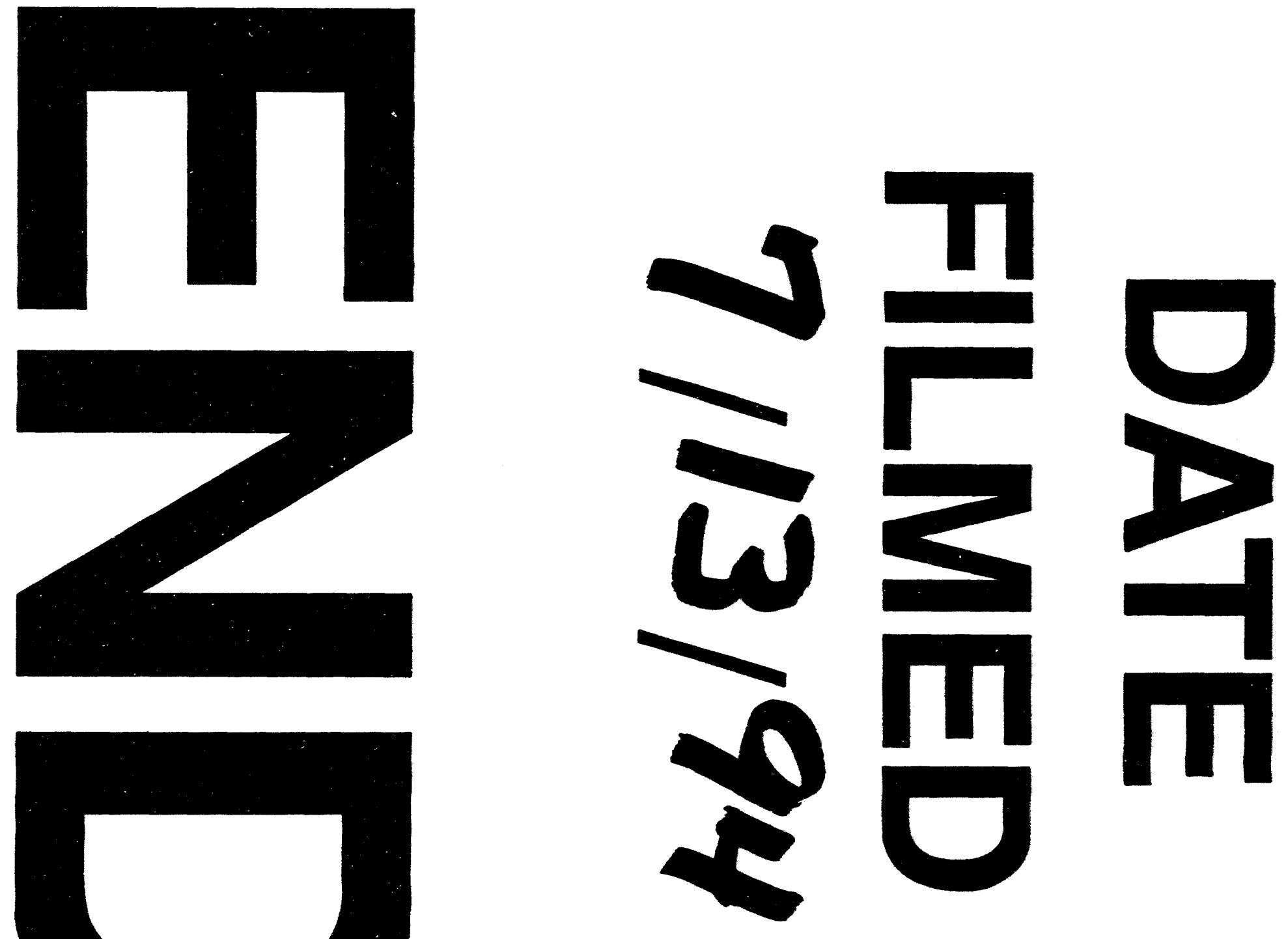

$<$

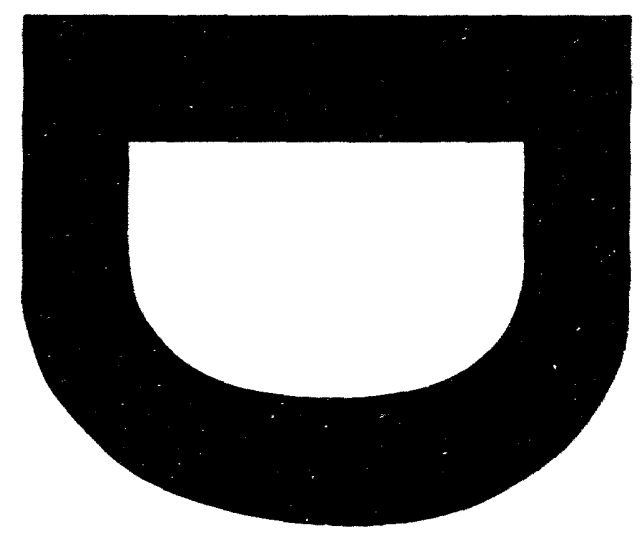




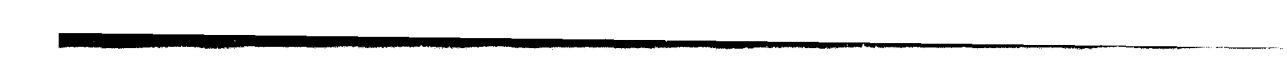

